\title{
Long non-coding RNAs in liver diseases: Focusing on nonalcoholic fatty liver disease, alcohol-related liver disease, and cholestatic liver disease
}

\author{
Sen Han ${ }^{1,2}$, Ting Zhang ${ }^{1}$, Praveen Kusumanchi', Nazmul Huda', Yanchao Jiang ${ }^{1}$, Zhihong Yang ${ }^{1}$, and \\ Suthat Liangpunsakul ${ }^{1,3,4}$ \\ 'Division of Gastroenterology and Hepatology, Department of Medicine, Indiana University School of Medicine, Indianapolis, IN, USA; \\ ${ }^{2}$ Key Laboratory of Carcinogenesis and Translational Research, Peking University Cancer Hospital, Beijing, China; ${ }^{3}$ Department of \\ Biochemistry and Molecular Biology, Indiana University School of Medicine, Indianapolis, IN; ${ }^{4}$ Roudebush Veterans Administration \\ Medical Center, Indianapolis, IN, USA
}

Long non-coding RNAs (IncRNAs), a class of transcribed RNA molecules with the lengths exceeding 200 nucleotides, are not translated into protein. They can modulate protein-coding genes by controlling transcriptional and posttranscriptional processes. The dysregulation of IncRNAs has been related to various pathological disorders. In this review, we summarized the current knowledge of IncRNAs and their implications in the pathogenesis of three common liver diseases: nonalcoholic fatty liver disease, alcohol-related liver disease, and cholestatic liver disease. Future studies to further define the role of IncRNAs and their mechanisms in various types of liver diseases should be explored. An improved understanding from these studies will provide us a useful perspective leading to mechanism-based intervention by targeting specific IncRNAs for the treatment of liver diseases. (Clin Mol Hepatol 2020;26:705-714)

Keywords: RNA, Long noncoding; Liver diseases; Etiology

\footnotetext{
Abbreviations:

ACC, acetyl coA carboxylase; ALD, alcohol-relatedliver disease; ATGL, adipose triglyceride lipase; BA, bile acid; BDL, bile duct ligation; CXCL5, C-X-C motif chemokine ligand 5; ECM, extracellular matrix; ED, excessive drinkers; EpCAM epithelial cell adhesion molecule; FASN, fatty acid synthase; FOXO1, Forkhead box protein O1; FXR, farnesoid X receptor; HC, healthy controls; HDACs, histone deacetylases; hnRNPA1, heterogeneous nuclear ribonucleoprotein A1; HSCs, hepatic stellate cells; IncARSR, long non-coding RNA activated in renal cell carcinoma with sunitinib resistance; IncRNA, long non-coding RNA; IncSHGL, long noncoding RNA suppressor of hepatic gluconeogenesis and lipogenesis; MALAT1, metastasis-associated lung adenocarcinoma transcript; $\mathrm{Mdr2}^{-1-}$, multidrug resistance 2 deficient; MEG3, maternally expressed gene 3; miRNA, microRNA; MLXIPL, MLX-interacting protein-like; MTOR, mammalian target of rapamycin; NAFLD, nonalcoholic fatty liver disease; NASH, nonalcoholic steatohepatitis; ncRNAs, non-coding RNAs; NEAT1, nuclear enriched abundant transcript 1; NTCP, sodium-taurocholate co-transporting polypeptide; PEPCK, phosphoenolpyruvate carboxykinase; PI3K, phosphatidylinositol 3-kinase; PTBP1, polypyrimidine tract-binding protein 1; SHP, small heterodimer partner; SRA, steroid receptor RNA activator; SREBP-1c, sterol regulatory element-binding protein 1; TC, total cholesterol; TG, triglyceride; ZEB1, zinc finger E-box binding homeobox 1
}

Corresponding author: Suthat Liangpunsakul

Division of Gastroenterology and Hepatology, Department of Medicine Indiana University School of Medicine, 702 Rotary Circle, Indianapolis, IN 46202, USA

Tel: +1-317-278-1630, Fax: +1-317-988-3180

E-mail: sliangpu@iu.edu

https://orcid.org/0000-0002-6504-8123 


\section{INTRODUCTION}

\section{Overview of long non-coding RNA (IncRNA)}

The central dogma "DNA-RNA-protein" indicates that key genetic information is stored in protein-coding genes; however, only a small percentage of the human transcriptome is translated into proteins, leaving the majority of the transcripts undefined. In recent decades, studies have focused on the remaining genome and found that up to $90 \%$ are non-coding RNAs (ncRNAs). ${ }^{1,2}$ These ncRNAs were initially considered as junk RNA or transcriptional noise; however, lately, they are involved in various cellular and disease processes, with emerging evidence of their interactions with each other as a complex regulatory network. ${ }^{3}$ In general, ncRNAs are divided into two groups depending on the length of the nucleotides, small ncRNAs (<200 nucleotides), and IncRNAs (>200 nucleotides). ${ }^{4}$

\section{Classifications of IncRNAs}

LncRNAs are transcribed by RNA polymerase II or III, mostly are 5'-capped, 3'-end poly-adenylated, and multi-exonic., 5 They exhibit poorer conservation than protein-coding genes. ${ }^{7}$ Most IncRNAs are folded after the transcription to form the tertiary structure, which impacts their functions. ${ }^{8,9}$ Based on their genomic location, IncRNAs are classified into the following categories; 1) intergenic IncRNA, located in between two protein-coding genes, transcribed in the same direction; 2) intronic IncRNA, located in the intronic regions and transcribed entirely from introns of a protein-coding gene; 3 ) sense IncRNA, transcribed from the same strand and the same direction as the surrounding proteincoding genes; they can be overlapping with exonic or intronic regions or cover the entire protein-coding sequence through an intron; 4) antisense IncRNA, transcribed from the opposite strand of surrounding protein-coding genes, also can be both exonic and intronic; and 5) bidirectional IncRNA, located within $1 \mathrm{~kb}$ of the promoter region of a protein-coding gene, but transcribed from the opposite strand. ${ }^{10,11}$ There are also some other criteria to classify IncRNAs based on their transcript properties, location in known genomic annotations, regulatory elements, and functions. ${ }^{12}$

\section{Functions of IncRNAs}

LncRNAs are involved in several biological processes such as the shaping of chromatin, replication, transcription, splicing, translation, and post-translational modification of proteins. ${ }^{13,14}$ For example, signal IncRNAs are only expressed at a specific time and localized to certain subcellular structures where they exert their functions. ${ }^{15}$ Decoy IncRNAs binds to regulatory factors such as transcription factor, RNA-binding protein, and chromatin modifier, thereafter altering their original biological activity. ${ }^{15}$ Guide InCRNAs regulate gene activation or repression by forming ribonucleoprotein complexes and mediating their localization to specific targets. ${ }^{15}$ Scaffold IncRNAs are similar to guide IncRNAs, but they affect the molecular component of the complex itself. ${ }^{16,17}$ Enhancer IncRNAs are produced from enhancer elements and influence the activation of target genes. ${ }^{18}$ Moreover, as a reservoir, some InCRNAs are processed to generate microRNAs (miRNAs). ${ }^{19,20}$ Some IncRNAs can bind miRNAs and function as a sponge for certain miRNAs to block their activity, which involves regulating the protein translation of the target mRNA. ${ }^{21,22}$ The main functions of the IncRNAs are shown in Figure 1.

Because IncRNAs are involved in a wide variety of important cellular processes, dysregulation of IncRNAs has been related to various pathological disorders. In this review, we summarized the current knowledge of IncRNAs and their implications in the pathogenesis of 3 common liver diseases, nonalcoholic fatty liver disease (NAFLD), alcohol-related liver disease (ALD), and cholestatic liver disease.

\section{A CRITICAL ROLE OF LnCRNAs IN NAFLD}

NAFLD comprises a spectrum of histopathological disorders ranging from simple steatosis, steatohepatitis, advanced fibrosis, and cirrhosis. ${ }^{23}$ Emerging studies have shown that IncRNAs are implicated in the pathogenesis of NAFLD through their roles in lipid and glucose metabolism.

\section{LncRNA steroid receptor RNA activator (SRA)}

SRA is involved in glucose and lipid metabolism. ${ }^{24,25}$ A study showed that the expression of liver adipose triglyceride lipase (ATGL) is induced in SRA knockout mice, and liver SRA and ATGL expression are inversely regulated under fasting conditions. ${ }^{25}$ Fork-head box protein 01 (FOX01) is a key transcription factor in the regulation of gluconeogenesis and the insulin response in the liver, which plays an important role in glucose homeostasis. ${ }^{26}$ F0X01 mainly targets insulin signaling and regulates metabolic 


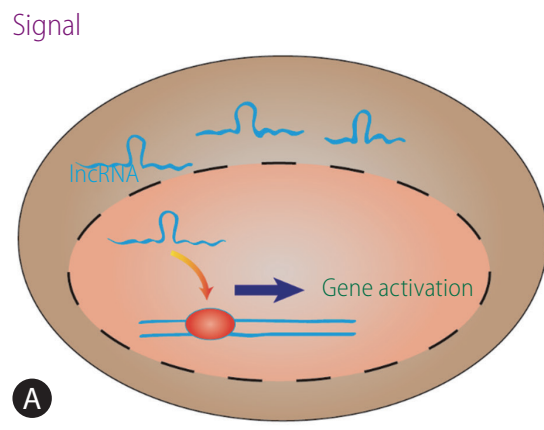

Decoy/sponge

Reservoir

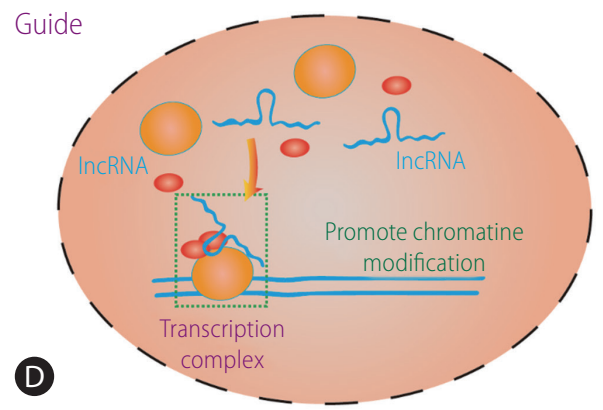

Scaffold
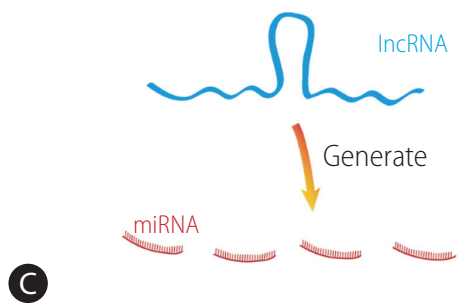

C

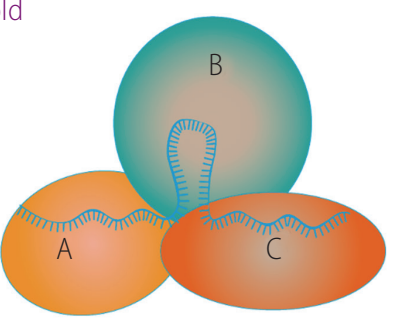

Enhancer

$\boldsymbol{F}$

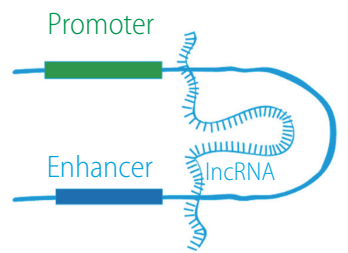

Figure 1. The main biological functions of IncRNA. (A) Signal IncRNA promotes gene expression. (B) Decoy IncRNA binds to regulatory factors and affects gene expression. LncRNA can sponge miRNA and affects its function. (C) Reservoir IncRNA generates miRNA. (D) Guide IncRNA takes ribonucleoprotein complexes to specific target. (E) Scaffold IncRNA combines other molecules to form complex. (F) Enhancer IncRNA increases target gene expression. IncRNA, long non-coding RNA; miRNA, microRNA.

homeostasis in response to oxidative stress, which also promotes the transcription of ATGL. By inhibiting the ability of FOXO1, InCRNA SRA inhibits ATGL promoter activity and regulates ATGL expression; a potential mechanism underlies NAFLD pathogenesis.

\section{LncRNA maternally expressed gene 3 (MEG3)}

LnCRNA MEG3 is involved in hepatic glucose metabolism. ${ }^{27}$ MEG3 is upregulated in mice fed with a high-fat diet, while knockdown of MEG3 remarkably abolishes hepatic triglyceride (TG) accumulation, up-regulates glycogen content, and promotes glucose tolerance. ${ }^{27}$ Palmitate, oleate or linoleate also increases MEG3 expression level in primary hepatocytes. Histone deacety- lases (HDACs) are a class of enzymes involved in expression of DNA, which catalyze the removal of acetyl groups from lysine residues in both histone and non-histone proteins. Knocking down of HDAC3 using siRNA or its inhibitor (RGFP966) significantly promotes MEG3 expression level, suggesting the role of histone acetylation in regulating its expression. ${ }^{27}$ Upregulation of MEG3 also increases FOXO1 expression and hepatic insulin resistance. ${ }^{28}$

\section{LncRNA activated in renal cell carcinoma with sunitinib resistance (IncARSR)}

The mRNA levels oflong non-coding RNA activated in renal cell carcinoma with sunitinib resistance (IncARSR) both in the serum 
and liver are significantly increased in NAFLD patients compared with the healthy controls (HC). ${ }^{29}$ Hepatic IncARSR is also induced in mice fed with methionine choline deficient diet. Overexpression of IncARSR enhances while knockdown of IncARSR ameliorates hepatic lipid accumulation in vivo and in vitro. Transient overexpression of IncARSR using AAV8-IncARSR virus markedly induces the expression of IncARSR and causes significant increase of intracellular TG contents in HepG2 cells. ${ }^{29}$ LncARSR overexpression also induces the expression of genes associated with lipogenesis, such as sterol regulatory element-binding protein 1 (SREBP-1C), fatty acid synthase (FASN), and stearoyl-CoA desaturase. Knockdown of SREBP-1c by short hairpin RNA is shown to block the effect of InCARSR on lipogenesis. In addition to SREBP-1C, overexpression of IncARSR also increases the level of phosphorylated Akt, and its effects was inhibited in the presence of LY294002, the phosphatidylinositol 3-kinase (PI3K) inhibitor. These data suggest the role of IncARSR-PI3K-Akt axis in regulating lipogenesis through SREBP-1c. ${ }^{29}$

LncARSR also modulates cholesterol metabolism in vitro and in vivo. ${ }^{30}$ LncARSR overexpression significantly elevated the serum total cholesterol (TC), low density lipoprotein cholesterol, and hepatic TC. The expression of genes related to cholesterol biosynthesis such as Srebp2, HMG-CoA reductase (Hmgcr), and HMGCoA synthase also increases, while the expression of Cyp7a1, the rate limiting enzyme of the conversion of cholesterol to bile acid (BA), decreases, suggesting the modulation of hepatic cholesterol biosynthesis by IncARSR. ${ }^{30}$ The effect of IncARSR on cholesterol biosynthesis is dependent on Akt/SREBP-2 pathway. ${ }^{30}$

\section{Lnc18q22.2}

Lnc18q22.2, a liver-specific IncRNA, is markedly induced in the liver tissue of nonalcoholic steatohepatitis (NASH) patients and correlated with NASH severity. ${ }^{31}$ The silencing of Inc18q22.2 expression results in reduced growth in $\mathrm{HepG} 2$ and immortalized human hepatocyte cells and promotes cell death in Huh7 and Hep3B cells; suggesting its crucial role for growth and viability of hepatocytes. ${ }^{31}$ Lnc18q22.2 is directly or indirectly involved in numerous essential biological processes in hepatocytes, including oxidation reduction, translation elongation, and regulation of cell death. Genes negatively regulated by $\operatorname{lnc} 18 q 22.2$ are enriched in the process of oxidation reduction; the finding which is consistent with the observation that NAFLD is often accompanied by increased oxidative stress. ${ }^{31}$ One of the most enriched pathways which is affected in the loss of Inc18q22.2 function is the regula- tion of cell death and apoptosis which may underlie the cell viability. Hepatocyte apoptosis is a major feature in NASH and in fact several anti-apoptosis genes were down-regulated after Inc18q22.2 knockdown, including MCL1, BCL2L1, BCL2L2, BFAR, CARD10, IGFIR, and MKL. ${ }^{31}$

\section{LncRNA suppressor of hepatic gluconeogenesis and lipogenesis (IncSHGL)}

The expression of IncSHGL in mouse and its human homologous InCRNA B4GALT1-AS1 are decreased in the liver of obese mice and NAFLD patients. ${ }^{32}$ Fasting hyperglycemia is improved the 4th and 7th day after the injection of adenovirus overexpressed IncSHGL in mice fed with high fat diet. Insulin tolerance test and hyperinsulinemic euglycemic clamp shows the improvement of global insulin resistance with the reduction in hepatic gluconeogenesis at the 7th day post injection. LncSHGL overexpression also reduces hepatic TG and serum insulin levels. Consistent with enhanced insulin sensitivity, hepatic IncSHGL overexpression repressed the lipolysis of white adipose tissue and reduced serum free fatty acid levels in high fat diet fed mice. ${ }^{32}$ LncSHGL overexpression also increases phosphorylated Akt with reduced protein levels of phosphoenolpyruvate carboxykinase, glucose 6-phosphatase, and FASN. Further mechanistic studies reveal that IncSHGL promotes Akt activation and FOXO1 nuclear exclusion in calmodulin-dependent manner. LncSHGL recruits heterogeneous nuclear ribonucleoprotein A1 to enhance calmodulin mRNA translation, activates the PI3K/Akt pathway, and inhibits the mammalian target of rapamycin (mTOR)/SREBP-1c pathway to prevent hepatic steatosis. $^{32}$

\section{Nuclear enriched abundant transcript 1 (NEAT1)}

LncRNA NEAT1 is involved in many diseases. As an oncogenic IncRNA, NEAT1 is remarkably up-regulated in lung cancer and breast cancer cells and promotes the breast cancer cell proliferation and DNA synthesis. ${ }^{33}$ NEAT1 also plays an important role in NAFLD pathogenesis. The levels of NEAT1 are increased in NAFLD in vivo and in vitro. ${ }^{34}$ Overexpression of NEAT1 increases both mRNA and protein level of acetyl coA carboxylase and FASN. It also leads to the induction of phosphorylated-mTOR and phosphorylated-p70S6K1. The effect of NEAT1 on acetyl co-A carboxylase (ACC) and FAS expression is ameliorated in the presence of rapamycin, an inhibitor of mTOR/S6K1 pathway. ${ }^{34}$ The inhibition of mTOR/S6K1 pathway mimics the effect of NEAT1 knockdown 
on the expression of ACC and FAS mRNA levels. Knocking down of NEAT1 significantly improves NAFLD phenotypes in the NAFLD rat model; suggesting the important role of the NEAT1/mTOR/ S6K1 axis in the pathogenesis of NAFLD. ${ }^{34}$

\section{Metastasis-associated lung adenocarcinoma transcript (MALAT1)}

MALAT1 plays an important role in diabetic complications and in the pathogenesis of diabetes-related microvascular disease and diabetic retinopathy. ${ }^{35}$ MALAT1 stimulates the production of inflammatory cytokine in the endothelial cells in the presence of high glucose. ${ }^{36}$ MALAT1 expression is dose-dependently increased in HepG2 cells and primary mouse hepatocytes when they are exposed to different doses of palmitate. ${ }^{37}$ The MALAT1 levels are also elevated in livers from ob/ob mice. ${ }^{37}$ Knockdown of MALAT1 significantly reduces the palmitate-induced TG and cholesterol accumulation in HepG2 cells and primary mouse hepatocytes. The reduction of MALAT1 expression abolishes palmitate-enhanced nuclear SREBP-1c protein level but had no effect on SREBP-1c precursor and SREBP-1c mRNA in HepG2 cells and mouse hepatocytes. MALAT1 knockdown also results in a significant decrease in the expression of SREBP-1c target genes. MALAT1 upregulates SREBP-1c expression by stabilizing SREBP-1c protein in hepatocytes by preventing ubiquitin-mediated degradation. ${ }^{37}$ Lastly, MALAT1 is also involved in NASH fibrosis. MALAT1 regulates C-XC motif chemokine ligand 5 (CXCL5) expression and the expression of both MALAT1 and CXCL5 is altered during the transition of hepatic stellate cells (HSCs) from a quiescent to activated state. $^{38}$

\section{LncRNA H19}

$\mathrm{H} 19$ as well as genes that are involved in hepatic lipogenesis are upregulated in mice fed with high fat diet. ${ }^{39,40} \mathrm{H} 19$ directly downregulates the expression of miR-130a which in turn inhibits the expression of peroxisome proliferator-activated receptor $\gamma$ level in the liver. ${ }^{39}$ It also promotes steatosis by regulating transcription factor MLX-interacting protein-like (MLXIPL) or carbohydrateresponsive element-binding protein. ${ }^{40} \mathrm{H} 19$ also serves as a lipid sensor by synergizing with RNA-binding protein, polypyrimidine tract-binding protein 1 (PTBP1), to modulate hepatic metabolic homeostasis. ${ }^{41}$ H19 can interact with PTBP1 to increase SREBP1 protein cleavage, stability, and transcriptional activity to activate lipogenesis genes. ${ }^{41}$

\section{A CRITICAL ROLE OF LnCRNAs IN ALD}

ALD occurs in a subset of patients with excessive alcohol intake. ${ }^{42,43}$ Similar to NAFLD, ALD also consists of a spectrum of pathological changes ranging from steatosis to fibrosis and cirrhosis. Although the relationship between alcohol consumption and liver disease is well established, severe alcohol-related morbidities develop in only a minority of people who consume alcohol in excess. Inter-individual differences in susceptibility to the toxic effects of alcohol have been extensively studied. ${ }^{43}$ Several new lines of evidence demonstrate the important role of IncRNAs in ALD pathogenesis

\section{MEG3}

Chronic alcohol consumption induced MEG3 expression in hepatocytes in both in vivo and in vitro models. Knocking down of MEG3 leads to a significant decrease in intracellular lipid accumulation in the presence of ethanol secondary to the inhibition of SREBP-1C and FASN. ${ }^{44}$ LncRNAs have been reported to act as decoys to sequester miRNAs to prevent them from binding to targets. ${ }^{45}$ There is a negative regulation between MEG3 and let-7c$5 p$, as MEG3 is a target of let-7c-5p in alcohol-induced liver injury. ${ }^{44}$ Overexpression of let-7c-5p Inhibits alcohol-induced steatosis by the regulation of its target gene NOD-like receptor family CARD domain containing $5{ }^{44}$

\section{LncRNAs AK128652 and AK054921}

Our group has conducted a study with the hypothesis that specific IncRNAs are critical for the progression from excessive drinkers (ED) without underlying liver disease to ALD and their unique role underpins the clinical observation on why only a subset of ED develop ALD. Using the IncRNA microarray global profiling, we identified unique IncRNA signature changes in $\mathrm{HC}$ and ED with more differentially expressed IncRNAs in patients with alcoholic cirrhosis. ${ }^{46}$ Detailed analysis of these IncRNAs in alcoholic cirrhosis stratified by Child-Pugh classes demonstrates 244 up-regulated and 181 down-regulated IncRNAs compared to HC. The number of uniquely up-regulated IncRNAs increased from 252 for those in Child-Pugh class A to 1,417 for those in Child-Pugh class $C$, whereas the number of uniquely down-regulated IncRNAs increased from 100 in those with Child class A to 1,594 for those in Child class $C{ }^{46}$ We reported 19 unique IncRNAs to be present in the liver on differential expression either $>2$ or $<2$ folds change. 


\section{CLNICAL and MOLECULAR}

\section{HEPATOLOGY}

Among them, IncRNA AK128652 exhibited the highest induction in patients with alcoholic cirrhosis followed by IncRNA AK054921. These two IncRNAs also exhibit prognostic significance on survival in patients with alcoholic cirrhosis; suggesting the unique roles of IncRNAs as potential prognostic biomarkers in patients with ALD. $^{46}$

\section{LncRNA Gm5091}

Hepatic fibrosis is a result of the dysregulation of the extracellular matrix (ECM) synthesis secondary to HSCs activation. HSCs activated by various toxic stimuli undergo proliferation and myofibroblastic transformation, lose their retinol and produce a considerable amount of ECM, such as a-smooth muscle actin and type I collagen. ${ }^{47,48}$ Several IncRNAs have been implicated in the pathogenesis of fibrosis and cirrhosis through their functions as either pro-fibrotic and anti-fibrotic effects. ${ }^{49-52}$ LncRNA Gm5091 is enriched in mouse HSC and significantly downregulated in the liver during alcohol-induced hepatic fibrosis. ${ }^{53}$ LncRNA Gm5091 negatively regulates cell migration, reactive oxygen species content, interleukin-1 $\beta$ secretion, and expression of collagen I and markers of HSC activation by sponging miR-27b/23b/24. ${ }^{53}$

\section{A CRITICAL ROLE OF LnCRNAs IN CHOLESTATIC LIVER DISEASE}

Cholestasis is a condition with a decrease in bile flow, either due to impaired secretion by hepatocytes or by obstruction. ${ }^{54}$ BAs, synthesized from cholesterol in the liver, play an essential role in eliminating cholesterol and facilitating dietary fat absorption. BA metabolism is strictly controlled by nuclear receptor signaling to coordinate BA synthetic enzymes and transporters. ${ }^{55}$ Accumulation of BAs in the hepatocytes can cause cell damage leading to inflammation and fibrosis. ${ }^{55}$ The nuclear receptor small heterodimer partner (SHP; NROB2) is an essential component in the negative feedback regulation of $B A$ synthesis. In response to the intrahepatic accumulation of $B A$, nuclear receptor farnesoid $X$ receptor (FXR) activates SHP to repress the expression of two regulatory enzymes sterol 12a-hydroxylase and cholesterol 7a-hydroxylase for de novo synthesis of BA. ${ }^{56,57}$ The activation of SHP also represses the transactivation of basolateral BA transporter of the

Table 1. Summary of IncRNAs and their roles in liver diseases

\begin{tabular}{|c|c|c|c|c|}
\hline LncRNA & Gene name & Targeted pathway & Related liver disease & Reference \\
\hline \multirow[t]{2}{*}{$\mathrm{H} 19$} & \multirow{2}{*}{$\begin{array}{l}\text { Imprinted maternally expressed } \\
\text { untranslated mRNA }\end{array}$} & Let-7 & Cholestatic liver disease & 59 \\
\hline & & $C C L-2, I L-6$ & Cholestatic liver disease & 66 \\
\hline LncARSR & $\begin{array}{l}\text { LncRNA regulator of Akt signaling } \\
\text { associated with HCC and RCC }\end{array}$ & SREBP-1C & NASH & 29 \\
\hline \multirow[t]{3}{*}{ MEG3 } & \multirow[t]{3}{*}{ MEG3 } & FOXO1 & NAFLD & 27 \\
\hline & & PTBP1/SHP & Cholestatic liver damage & 50 \\
\hline & & miR-7-5p & ALD & 44 \\
\hline MALAT1 & MALAT1 & SREBP-1C & NAFLD & 37,51 \\
\hline SRA & Steroid receptor coactivator & FOX01/ATGL & NAFLD & 25 \\
\hline NEAT1 & NEAT1 & Unclear & NAFLD & 34 \\
\hline LncSHGL & LncSHGL & mTOR/SREBP-1c & NAFLD & 32 \\
\hline Lnc18q22.2 & - & Unclear & NASH & 31 \\
\hline AK054921 & - & Unclear & ALD & 46 \\
\hline AK128652 & - & Unclear & ALD & 46 \\
\hline Gm5091 & - & miR-27b/23b/24 & Alcoholic cirrhosis & 53 \\
\hline
\end{tabular}

IncRNA, long non-coding RNA; IL, interleukin; IncARSR, long non-coding RNA activated in renal cell carcinoma with sunitinib resistance; HCC, hepatocellular carcinoma; RCC, renal cell carcinoma; SREBP-1c, sterol regulatory element-binding protein 1; NASH, nonalcoholic steatohepatitis; MEG3, maternally expressed gene 3; FOXO1, Fork-head box protein 01; PTBP1, polypyrimidine tract-binding protein 1; SHP, small heterodimer partner; NAFLD, nonalcoholic fatty liver disease; ALD, alcohol-related liver disease; MALAT1, metastasis-associated lung adenocarcinoma transcript; SRA, steroid receptor RNA activator; ATGL, adipose triglyceride lipase; NEAT1, nuclear enriched abundant transcript 1; InCSHGL, long non-coding RNA suppressor of hepatic gluconeogenesis and lipogenesis; mTOR, mammalian target of rapamycin. 
hepatocytes such as sodium-taurocholate co-transporting polypeptide (NTCP) to block uptake of BA. ${ }^{58}$ Recent studies suggest the role of IncRNAs in cholestasis.

\section{LncRNA H19}

In the bile duct ligation (BDL) model, the hepatic let-7 family is markedly induced by $\mathrm{H} 19 .^{59} \mathrm{H} 19$ promotes the biogenesis and expression of a cluster of let-7 miRNAs, including let-7a-5p, let-7d$5 p$, and let-7f-5p, in hepatic cholestasis and functionally suppresses their bioavailability. $\mathrm{H} 19$ antagonizes the expression of its binding partner, PTBP1, which associates with pre-let-7d and prelet-7a-1 and inhibits let-7 biogenesis but promotes their bioavailability. ${ }^{59} \mathrm{H} 19$ facilitates hepatic cholestasis by preventing zinc finger E-box binding homeobox 1 (ZEB1) mediated inhibition of epithelial cell adhesion molecule (EPCAM). ZEB1 is a transcriptional repressor that plays a role in epithelial-to-mesenchymal transition during fibrogenesis. ${ }^{60} \mathrm{H} 19$ induces EpCAM but inhibits ZEB1 expression in the BDL model and overexpression of ZEB1 or knockdown of EpCAM ameliorates H19-induced cholestasis. ${ }^{61}$

In another model of cholestasis using multidrug resistance 2 deficient $\left(\mathrm{Mdr}^{-1-}\right.$ ) mice, the expression of hepatic H19 in 100-daysold wild-type mice was very low with a slight but significant increase in male $\mathrm{Mdr}^{-1-}$ mice. However, there was a markedly increase in its expression by 200-folds in female $\mathrm{Mdr}^{-/-}$mice with more severe cholestasis. ${ }^{62}$ Further mechanistic experiments revealed that taurocholate and $17 \beta$-estradiol up-regulate H19 expression and that knocking down $\mathrm{H} 19$ ameliorates cholestatic liver injury in female $\mathrm{Mdr}^{-/-}$mice. ${ }^{62}$

H19 is also implicated in biliary atresia. ${ }^{63}$ Hepatic H19 expression and the levels of serum H19 are associated with the degree of fibrosis in patients with biliary atresia. ${ }^{63}$ Additionally, its expression is correlated with the up-regulation of hepatic sphingosine 1-phosphate receptor 2 and sphingosine kinase 2 which are known to attribute to cholestatic liver injury by enhancing cholangiocyte proliferation and inflammatory responses..$^{63-65}$

\section{MEG3}

MEG3 can interact with PTBP1 as identified using RNA pulldown with biotin-labeled sense or anti-sense MEG3RNA followed by mass spectrometry. Forced expression of MEG3 in hepatocellular carcinoma cells guided and facilitated PTBP1 binding to SHP coding region, resulting in SHP mRNA decay. Transient overexpression of MEG3RNA in vivo in mouse liver caused rapid SHP
mRNA degradation and cholestatic liver injury, which was accompanied by the disruption of BA homeostasis, the elevation of liver enzymes, and dysregulation of BA synthetic enzymes and metabolic genes. ${ }^{50}$ Summary of IncRNAs and their roles in liver diseases are shown in Table 1.

\section{PERSPECTIVES AND CONCLUSION}

Numerous IncRNAs are implicated in the pathogenesis of NAFLD, ALD, and cholestatic liver diseases. The regulatory network of IncRNAs and the underlying mechanisms leading to liver diseases are complicated. Given a large number of IncRNAs and the extensive studies in this area, new IncRNAs with relevance to liver diseases are likely to be discovered. While the field is advancing, there is a critical need to translate the observations in cell lines or animal models and determine if the functions of these IncRNAs recapitulate the disease pathogenesis in humans. Detection of circulating IncRNAs raises the potential for their use as the biomarkers for risk prediction, diagnostic applicability, or predictors of prognosis in liver diseases. It will be of interest to determine the use of specific IncRNAs that can perhaps supplement and eventually replace invasive liver biopsy for diagnostic and prognostic purposes. Modulation of specific IncRNAs especially in mouse models has a profound impact on the liver disease phenotypes, suggesting the therapeutic potentials of IncRNA-based therapy. Despite the considerable promise, there are several challenges on the safety and the potential off-target effects. An improved understanding and knowledge resulting from the intensive studies of IncRNAs in liver diseases will unquestionably provide useful perspectives and generate new information that will eventually translate to novel clinical applications.

\section{Authors' contribution}

SH: Literature review, SH, TZ, YJ: Drafting the manuscript, PK, $\mathrm{NH}, \mathrm{SL}$, and ZY: A critical review of the manuscript, SH and SL: Finalizing the manuscript. All authors have read and approved the manuscript for submission.

\section{Acknowledgements}

ZY is supported by NIH K01AA26385, Indiana University Research Support Fund Grant (IU RSFG), and the Ralph W. and Grace M. Showalter Research Trust and the Indiana Institute for Medical Research; SL is supported in part by R01 DK107682, R01 AA025208, U01 AA026917, UH2/UH3 AA026903, VA Merit 
Award 1101CX000361 and Indiana Clinical and Translational Sciences Institute, UL1TR002529, National Center for Advancing Translational Sciences, Clinical and Translational Sciences Award, Showalter Scholar Indiana University School of Medicine, and IUSM Dean's Scholar in Medical Research; PK is supported by the grant from Indiana Institute for Medical Research (IIMR).

\section{Conflicts of Interest}

The authors have no conflicts to disclose.

\section{REFERENCES}

1. ENCODE Project Consortium, Birney E, Stamatoyannopoulos JA, Dutta A, Guigó R, Gingeras TR, et al. Identification and analysis of functional elements in $1 \%$ of the human genome by the ENCODE pilot project. Nature 2007;447:799-816.

2. ENCODE Project Consortium. An integrated encyclopedia of DNA elements in the human genome. Nature 2012;489:57-74.

3. Yamamura S, Imai-Sumida M, Tanaka Y, Dahiya R. Interaction and cross-talk between non-coding RNAs. Cell Mol Life Sci 2018;75:467484.

4. Djebali S, Davis CA, Merkel A, Dobin A, Lassmann T, Mortazavi A, et al. Landscape of transcription in human cells. Nature 2012;489:101108.

5. Erdmann VA, Szymanski M, Hochberg A, de Groot N, Barciszewski J. Collection of mRNA-like non-coding RNAs. Nucleic Acids Res 1999:27:192-195.

6. Danko CG, Hah N, Luo X, Martins AL, Core L, Lis JT, et al. Signaling pathways differentially affect RNA polymerase II initiation, pausing, and elongation rate in cells. Mol Cell 2013;50:212-222.

7. Johnsson P, Lipovich L, Grandér D, Morris KV. Evolutionary conservation of long non-coding RNAs; sequence, structure, function. Biochim Biophys Acta 2014;1840:1063-1071.

8. Novikova IV, Hennelly SP, Sanbonmatsu KY. Tackling structures of long noncoding RNAs. Int J Mol Sci 2013;14:23672-23684.

9. Yan K, Arfat Y, Li D, Zhao F, Chen Z, Yin C, et al. Structure prediction: new insights into decrypting long noncoding RNAs. Int J Mol Sci 2016;17:132.

10. Dhanoa JK, Sethi RS, Verma R, Arora JS, Mukhopadhyay CS. Long non-coding RNA: its evolutionary relics and biological implications in mammals: a review. J Anim Sci Technol 2018;60:25.

11. Hermans-Beijnsberger $S$, van Bilsen $M$, Schroen B. Long non-coding RNAs in the failing heart and vasculature. Noncoding RNA Res 2018;3:118-130.

12. Jarroux J, Morillon A, Pinskaya M. History, Discovery, and Classification of IncRNAs. Adv Exp Med Biol 2017;1008:1-46.
13. Huang B, Zhang R. Regulatory non-coding RNAs: revolutionizing the RNA world. Mol Biol Rep 2014;41:3915-3923.

14. Dykes IM, Emanueli C. Transcriptional and post-transcriptional gene regulation by long non-coding RNA. Genomics Proteomics Bioinformatics 2017;15:177-186.

15. Wang KC, Chang HY. Molecular mechanisms of long noncoding RNAs. Mol Cell 2011;43:904-914.

16. Guttman M, Donaghey J, Carey BW, Garber M, Grenier JK, Munson $G$, et al. lincRNAs act in the circuitry controlling pluripotency and differentiation. Nature 2011;477:295-300.

17. Tsai MC, Manor O, Wan Y, Mosammaparast N, Wang JK, Lan F, et al. Long noncoding RNA as modular scaffold of histone modification complexes. Science 2010;329:689-693.

18. Long $Y$, Wang $X$, Youmans DT, Cech TR. How do IncRNAs regulate transcription? Sci Adv 2017;3:eaao2110.

19. Keniry A, Oxley D, Monnier P, Kyba M, Dandolo L, Smits G, et al. The $\mathrm{H} 19$ lincRNA is a developmental reservoir of miR-675 that suppresses growth and Igf1r. Nat Cell Biol 2012;14:659-665.

20. Yoon JH, Abdelmohsen K, Gorospe M. Functional interactions among microRNAs and long noncoding RNAs. Semin Cell Dev Biol 2014;34:9-14.

21. Salmena L, Poliseno L, Tay Y, Kats L, Pandolfi PP. A ceRNA hypothesis: the Rosetta Stone of a hidden RNA language? Cell 2011;146: 353-358.

22. Ebert MS, Sharp PA. MicroRNA sponges: progress and possibilities. RNA 2010;16:2043-2050.

23. Streba LA, Vere CC, Rogoveanu I, Streba CT. Nonalcoholic fatty liver disease, metabolic risk factors, and hepatocellular carcinoma: an open question. World J Gastroenterol 2015;21:4103-4110.

24. Zhao Y, Wu J, Liangpunsakul S, Wang L. Long non-coding RNA in liver metabolism and disease: current status. Liver Res 2017;1:163167.

25. Chen G, Yu D, Nian X, Liu J, Koenig RJ, Xu B, et al. LncRNA SRA promotes hepatic steatosis through repressing the expression of adipose triglyceride lipase (ATGL). Sci Rep 2016;6:35531.

26. Puigserver $P$, Rhee J, Donovan J, Walkey CJ, Yoon JC, Oriente F, et al. Insulin-regulated hepatic gluconeogenesis through FOX01-PGC1alpha interaction. Nature 2003;423:550-555.

27. Zhu X, Wu YB, Zhou J, Kang DM. Upregulation of IncRNA MEG3 promotes hepatic insulin resistance via increasing Fox01 expression. Biochem Biophys Res Commun 2016;469:319-325.

28. Zhu X, Li H, Wu Y, Zhou J, Yang G, Wang W. IncRNA MEG3 promotes hepatic insulin resistance by serving as a competing endogenous RNA of miR-214 to regulate ATF4 expression. Int J Mol Med 2019:43:345-357.

29. Zhang M, Chi X, Qu N, Wang C. Long noncoding RNA IncARSR promotes hepatic lipogenesis via Akt/SREBP-1c pathway and contributes to the pathogenesis of nonalcoholic steatohepatitis. Biochem 
Biophys Res Commun 2018;499:66-70.

30. Huang J, Chen S, Cai D, Bian D, Wang F. Long noncoding RNA InCARSR promotes hepatic cholesterol biosynthesis via modulating Akt/SREBP-2/HMGCR pathway. Life Sci 2018;203:48-53.

31. Atanasovska B, Rensen SS, van der Sijde MR, Marsman G, Kumar $V$, Jonkers I, et al. A liver-specific long noncoding RNA with a role in cell viability is elevated in human nonalcoholic steatohepatitis. Hepatology 2017;66:794-808.

32. Wang J, Yang W, Chen Z, Chen J, Meng Y, Feng B, et al. Long noncoding RNA IncSHGL recruits hnRNPA1 to suppress hepatic gluconeogenesis and lipogenesis. Diabetes 2018;67:581-593.

33. Qian K, Liu G, Tang Z, Hu Y, Fang Y, Chen Z, et al. The long noncoding RNA NEAT1 interacted with miR-101 modulates breast cancer growth by targeting EZH2. Arch Biochem Biophys 2017;615:1-9.

34. Wang $X$. Down-regulation of IncRNA-NEAT1 alleviated the nonalcoholic fatty liver disease via mTOR/S6K1 signaling pathway. J Cell Biochem 2018;119:1567-1574.

35. Yan B, Tao ZF, Li XM, Zhang H, Yao J, Jiang Q. Aberrant expression of long noncoding RNAs in early diabetic retinopathy. Invest Ophthalmol Vis Sci 2014;55:941-951.

36. Puthanveetil P, Chen S, Feng B, Gautam A, Chakrabarti S. Long noncoding RNA MALAT1 regulates hyperglycaemia induced inflammatory process in the endothelial cells. J Cell Mol Med 2015;19:14181425.

37. Yan C, Chen J, Chen N. Long noncoding RNA MALAT1 promotes hepatic steatosis and insulin resistance by increasing nuclear SREBP1c protein stability. Sci Rep 2016;6:22640.

38. Leti F, Legendre C, Still CD, Chu X, Petrick A, Gerhard GS, et al. Altered expression of MALAT1 IncRNA in nonalcoholic steatohepatitis fibrosis regulates CXCL5 in hepatic stellate cells. Transl Res 2017;190:25-39.e21.

39. Liu J, Tang T, Wang GD, Liu B. LncRNA-H19 promotes hepatic lipogenesis by directly regulating miR-130a/PPARy axis in non-alcoholic fatty liver disease. Biosci Rep 2019;39:BSR20181722.

40. Wang H, Cao Y, Shu L, Zhu Y, Peng Q, Ran L, et al. Long non-coding RNA (InCRNA) H19 induces hepatic steatosis through activating MLXIPL and mTORC1 networks in hepatocytes. J Cell Mol Med 2020;24:1399-1412.

41. Liu C, Yang Z, Wu J, Zhang L, Lee S, Shin DJ, et al. Long noncoding RNA H19 interacts with polypyrimidine tract-binding protein 1 to reprogram hepatic lipid homeostasis. Hepatology 2018;67:1768-1783.

42. Rehm J, Samokhvalov AV, Shield KD. Global burden of alcoholic liver diseases. J Hepatol 2013;59:160-168.

43. Liangpunsakul S, Haber P, McCaughan GW. Alcoholic liver disease in Asia, Europe, and North America. Gastroenterology 2016;150:17861797.

44. Wang Q, Li M, Shen Z, Bu F, Yu H, Pan X, et al. The long non-coding RNA MEG3/miR-let-7c-5p axis regulates ethanol-induced hepatic steatosis and apoptosis by targeting NLRC5. Front Pharmacol 2018;9:302.

45. Quinn JJ, Chang HY. Unique features of long non-coding RNA biogenesis and function. Nat Rev Genet 2016;17:47-62.

46. Yang Z, Ross RA, Zhao S, Tu W, Liangpunsakul S, Wang L. LncRNA AK054921 and AK128652 are potential serum biomarkers and predictors of patient survival with alcoholic cirrhosis. Hepatol Commun 2017;1:513-523.

47. Lee YS, Jeong WI. Retinoic acids and hepatic stellate cells in liver disease. J Gastroenterol Hepatol 2012;27 Suppl 2:75-79.

48. Tsuchida T, Friedman SL. Mechanisms of hepatic stellate cell activation. Nat Rev Gastroenterol Hepatol 2017;14:397-411.

49. He Z, Yang D, Fan $X$, Zhang $M$, Li Y, Gu X, et al. The roles and mechanisms of IncRNAs in liver fibrosis. Int J Mol Sci 2020;21:1482.

50. Zhang L, Yang Z, Trottier J, Barbier O, Wang L. Long noncoding RNA MEG3 induces cholestatic liver injury by interaction with PTBP1 to facilitate shp mRNA decay. Hepatology 2017;65:604-615.

51. Yu F, Lu Z, Cai J, Huang K, Chen B, Li G, et al. MALAT1 functions as a competing endogenous RNA to mediate Rac1 expression by sequestering miR-101b in liver fibrosis. Cell Cycle 2015;14:3885-3896.

52. Jiang $X$, Ning Q. The mechanism of IncRNA H19 in fibrosis and its potential as novel therapeutic target. Mech Ageing Dev 2020;188: 111243.

53. Zhou B, Yuan W, Li X. LncRNA Gm5091 alleviates alcoholic hepatic fibrosis by sponging miR-27b/23b/24 in mice. Cell Biol Int 2018;42:1330-1339.

54. Goldstein J, Levy C. Novel and emerging therapies for cholestatic liver diseases. Liver Int 2018;38:1520-1535.

55. Chiang JY. Recent advances in understanding bile acid homeostasis. F1000Res 2017;6:2029.

56. Goodwin B, Jones SA, Price RR, Watson MA, McKee DD, Moore LB, et al. A regulatory cascade of the nuclear receptors FXR, SHP-1, and LRH-1 represses bile acid biosynthesis. Mol Cell 2000;6:517-526.

57. Zhang Y, Hagedorn CH, Wang L. Role of nuclear receptor SHP in metabolism and cancer. Biochim Biophys Acta 2011;1812:893-908.

58. Denson LA, Sturm E, Echevarria W, Zimmerman TL, Makishima M, Mangelsdorf DJ, et al. The orphan nuclear receptor, shp, mediates bile acid-induced inhibition of the rat bile acid transporter, ntcp. Gastroenterology 2001;121:140-147.

59. Zhang L, Yang Z, Huang W, Wu J. H19 potentiates let-7 family expression through reducing PTBP1 binding to their precursors in cholestasis. Cell Death Dis 2019;10:168.

60. Gregory PA, Bert AG, Paterson EL, Barry SC, Tsykin A, Farshid $G$, et al. The miR-200 family and miR-205 regulate epithelial to mesenchymal transition by targeting ZEB1 and SIP1. Nat Cell Biol 2008;10:593-601.

61. Song Y, Liu C, Liu X, Trottier J, Beaudoin M, Zhang L, et al. H19 promotes cholestatic liver fibrosis by preventing ZEB1-mediated inhibi- 


\section{CLINICAL and MOLECULAR}

tion of epithelial cell adhesion molecule. Hepatology 2017;66:11831196.

62. Li X, Liu R, Yang J, Sun L, Zhang L, Jiang Z, et al. The role of long noncoding RNA H19 in gender disparity of cholestatic liver injury in multidrug resistance 2 gene knockout mice. Hepatology 2017;66:869-884

63. Xiao Y, Liu R, Li X, Gurley EC, Hylemon PB, Lu Y, et al. Long Noncoding RNA H19 contributes to cholangiocyte proliferation and cholestatic liver fibrosis in biliary atresia. Hepatology 2019;70:1658-1673.

64. Nagahashi M, Takabe K, Liu R, Peng K, Wang X, Wang Y, et al. Conjugated bile acid-activated S1P receptor 2 is a key regulator of sphingosine kinase 2 and hepatic gene expression. Hepatology 2015;61:1216-1226.

65. Liu R, Zhao R, Zhou X, Liang X, Campbell DJ, Zhang X, et al. Conjugated bile acids promote cholangiocarcinoma cell invasive growth through activation of sphingosine 1-phosphate receptor 2. Hepatology 2014;60:908-918.

66. Li X, Liu R, Wang Y, Zhu W, Zhao D, Wang X, et al. CholangiocyteDerived Exosomal IncRNA H19 Promotes Macrophage Activation and Hepatic Inflammation under Cholestatic Conditions. Cells 2020;9:190. 